\title{
Repetitive transcranial magnetic stimulation improves neurological function after cerebral ischemia in rats by increasing CREB-regulated TrkB via activation of cAMP/PKA and Ca2+/CaMKIV signaling pathways
}

\section{Lisha Chang}

North China University of Science and Technology Affiliated Hospital

\section{Zhaowang An}

North China University of Science and Technology Affiliated Hospital

Jiang Zhang

North China University of Science and Technology Affiliated Hospital

Fuling Zhou

North China University of Science and Technology Affiliated Hospital

\section{Dali Wang}

North China University of Science and Technology Affiliated Hospital

Jian Liu

North China University of Science and Technology Affiliated Hospital

Yunhe Zhang ( $\boldsymbol{\sim}$ zhangyunhezyh@yeah.net)

North China University of Science and Technology Affiliated Hospital

\section{Research}

Keywords: Cerebral ischemia, Neurological function, Repetitive transcranial magnetic stimulation, cAMPresponse element binding protein, cAMP/PKA pathway, Ca2+/CaMKIV pathway, Tropomyosin-related kinase B

Posted Date: November 11th, 2019

DOI: https://doi.org/10.21203/rs.2.17132/v1

License: (c) (1) This work is licensed under a Creative Commons Attribution 4.0 International License.

Read Full License 


\section{Abstract}

Background: Cerebral ischemia is the most prevalent form of clinical stroke. Repetitive transcranial magnetic stimulation (rTMS) can modulate excitability of the cerebral cortex, and this effect is maintained after the stimulation is terminated. However, the underlying mechanisms of rTMS in cerebral ischemia remain unclear.

Methods: Herein, we identified the effect of rTMS on cerebral ischemia and further explored the underlying mechanisms. An in vitro model was established using primary cultured neurons under conditions of oxygen-glucose deprivation (OGD), followed by $1 \mathrm{~Hz}$ or $10 \mathrm{~Hz}$ rTMS treatment. The levels of CREB, PKA and CaMKIV were depleted in neurons to explore the underlying regulatory mechanisms of TrkB by rTMS via CREB. A rat model of cerebral ischemia was established by middle cerebral artery occlusion (MCAO) and the rats were treated with $1 \mathrm{~Hz}$ or $10 \mathrm{~Hz}$ rTMS to investigate the effect of rTMS on neurobehavior, CREB expression, and cAMP/PKA and Ca 2+ /CaMKIV pathways.

Results: rTMS was observed to promote nerve recovery ability in rats with cerebral ischemia, which was accompanied by high expression of TrkB. In OGD-treated neurons, rTMS activated CREB by upregulating CAMP/PKA and Ca 2+ /CaMKIV pathways. Moreover, rTMS induced the activation of CREB to upregulate TrkB. In MCAO rats, rTMS increased the CREB expression, enhanced CAMP, PKA and CaMKIV phosphorylation, and promoted the binding of CREB to TrkB.

Conclusions: Taken together, rTMS upregulated CREB and TrkB to improve neurological function in rats with cerebral ischemia by activating cAMP/PKA and Ca 2+ /CaMKIV pathways, which could be of great significance for cerebral ischemia therapy.

\section{Background}

Cerebral ischemia is the most common stroke inducer, which is characterized by reduced blood flow to brain tissues, causing insufficient transport of glucose, oxygen and other nutrients, and resulting in metabolic changes and possibly cell death [1]. Ischemia usually causes debilitating neurologic deficits which seriously influence the quality of life of patients [2]. Ischemic stroke triggers severe inflammatory responses related to both adaptive and innate immunity [3]. Neurological deficits induced by cerebral ischemia reperfusion injury were frequently detected after the blood flow was restored in patients with cerebral stroke [4]. Although the pharmacological agents and intervention techniques have achieved improvements, there is still a lack of proficient techniques and drugs to deal with its post-rehabilitation or recovery [5]. Recently, repetitive transcranial magnetic stimulation (rTMS) as a therapeutic approach for functional recovery of subacute cerebral ischemia has been linked to an anti-apoptotic mechanism in the peri-ischemic area [6].

rTMS is a non-invasive brain stimulation which is acceptable to most patients. Its ability to regulate and maintain cerebral cortex excitability until stimulation is terminated to be of great clinical significance [7]. In a previous study, rTMS of human-derived neuron-like cells elevated intracellular cAMP levels and 
promoted cAMP-response element binding protein (CREB) phosphorylation [8]. CREB, which is downstream of various signaling cascades and in the center of multiple kinase pathways driven by activity, could regulate the intrinsic excitability of the neuron and is closely associated with learning and memory abilities [9]. Additionally, CREB triggers the expression of some neuroprotective proteins such as brain-derived neurotrophic factor (BDNF), which facilitates the survival of neurons after ischemia [10]. The activation of CREB regulates the induction of various genes involving BDNF, which serves as a promoter primarily by binding to tropomyosin-related kinase $B$ (TrkB) [11]. As a component of Trk family, TrkB functioned as a receptor tyrosine kinase for BDNF, and both TrkB and BDNF are essential to the nervous system development [12]. Low frequency magnetic stimulation modulates the synaptic plasticity of hippocampal neurons by activating the BDNF-TrkB pathway [13]. A previous study has shown that the activation of the BDNF-TrkB-PI3K/Akt pathway was associated with reduced cell apoptosis in the focal cerebral ischemia [14]. Interestingly, the activation of cAMP/PKA pathway was linked to the brain vascular endothelial cell proliferation after ischemic insult in vitro [15]. Under neuronal stimulation, CaMKIV activation is capable of inducing synaptic modifications and transcriptional responses, where both of them could affect cognitive and emotional behavior [16]. Based on the above research, rTMS may affect the neurological function after cerebral ischemia in rats through regulating CREB and TrkB. The current study was conducted to investigate the role of rTMS in activating CREB, and the regulation of TrkB in the neurological function of rats with cerebral ischemia. Meanwhile, different pathways such as cAMP/PKA and $\mathrm{Ca}^{2+} / \mathrm{CaMKIV}$ were further explored to offer a better understanding for the underlying molecular mechanisms in development of cerebral ischemia.

\section{Materials And Methods}

\section{Ethics statement}

The animal experiments were performed in strict accordance with the recommendations in the Guide for the Care and Use of Laboratory Animals of the National Institutes of Health. The protocol was approved by the Animal Ethics Committee of North China University of Science and Technology Affiliated Hospital.

\section{Study subjects}

A total of 30 adult male Sprague Dawley (SD) rats (SCXK [Xiang] 2009-0004, aged 8 - 10 weeks, weighing $200-220 \mathrm{~g}$ ) at specific pathogen free (SPF) grade were provided by Hunan SLAC Laboratory Animal Co., Ltd. (Changsha, Hunan, China). The rats were allowed free access to food and water with alternate periods of $12 \mathrm{~h}$ with and without light. Five rats were regarded as the mock, another 5 were subjected to sham operation and the remaining 20 rats were used for the model establishment. 
The adenoviral vector was constructed in 293T cells, using the adenovirus expression vector kit (Takara, Tokyo, Japan). The recombinant adenovirus was obtained at passage 1. Following removal of wild type (WT) virus from 293T cells by differential infection method, adenovirus with a higher titer was collected at passage 4 for later use. siRNA negative control (siNC), siRNA targeting CREB (siCREB), siRNA targeting PKA (siPKA), and siRNA targeting CaMKIV (siCaMKIV) were purchased from Guangzhou Ruibo Biotechnology Co., Ltd. (Guangzhou, Guangdong, China) and packed with the use of adenovirus.

\section{Development of a cell model with OGD and cell transfection}

The cerebral cortex, striatum and hippocampus were separated from 24-hour-old neonatal rats, washed 3 times in D-Hanks solution, and cut into pieces. The pieces were detached with $2.5 \mathrm{~g} / \mathrm{L}$ trypsin at $37^{\circ} \mathrm{C}$ for $10 \mathrm{~min}$. After the detachment was terminated by Dulbecco's modified eagle medium (DMEM) containing $10 \%$ fetal bovine serum (FBS), the pieces were centrifuged at $178 \times \mathrm{g}$ for $10 \mathrm{~min}$. The stop buffer was removed and the steps were repeated once. The filtrate was then passed through the sieve and centrifuged at $178 \times \mathrm{g}$ for $10 \mathrm{~min}$, followed by two washes with D-Hanks solution. Single cell suspensions were prepared by adding DMEM containing $10 \%$ bovine serum albumin (BSA) and $10 \%$ B-27, and cell density was adjusted to $2 \times 10^{5} \mathrm{cells} / \mathrm{mL}$. Cells were then plated in 96-well culture plates pre-coated with $0.05 \mathrm{~g} / \mathrm{L}$ Poly-L-Lysin and cultured at $37^{\circ} \mathrm{C}$ in a $5 \% \mathrm{CO}_{2}$ incubator. The neurons were cultured in an oxygen-glucose deprivation (OGD) environment (sugar-free DMEM, $5 \% \mathrm{CO}_{2}, \mathrm{CO}_{2}<1 \%$ and $95 \% \mathrm{~N}_{2}$ ) for I $\mathrm{h}$ every $48 \mathrm{~h}$ to prepare single OGD cortical neurons. In order to simulate the model of recurrent ischemia in vivo, two OGD cortical neurons were required to be produced. After receiving the first OGD treatment for $60 \mathrm{~min}$, neurons were cultured with the conventional culture medium under normal culture conditions for $3 \mathrm{~h}$, and then subjected to OGD treatment for $60 \mathrm{~min}$. After that, the neurons were conventionally cultured. Prior to transfection with adenovirus expressing siNC, siCREB, siPKA and siCaMKIV, primary neurons were seeded in 6-well plates for $48 \mathrm{~h}$. When cell confluence reached $70-80 \%$, they were transfected according to the instructions described in lipofectamine 2000 (11668-019, Invitrogen, Carlsbad, CA, USA) and cultured for 24 - $48 \mathrm{~h}$ for subsequent experiments.

\section{Establishment of middle cerebral artery occlusion (MCAO)-induced cerebral ischemia model in rats}

Focal cerebral ischemia model was induced by MCAO as previously described $[17,18]$. General anesthesia was induced with $3 \%$ pentobarbital sodium (P3761, Sigma-Aldrich Chemical Company, St Louis, MO, USA) and anesthesia was maintained by inhalation of $1.5 \%$ halothane. The rats were partially sterilized, and their skin was cut at the midpoint of the line connecting the left eye and left ear under the microscope. The skin, fascia and salivary glands of the rats were separated and a bipolar coagulator was used to coagulate the blood vessels. The fascia was separated to the tibia, the left tibia was cut, and the muscle tissue was separated to the base of the skull. The skull was removed with a micro-bone drill. The residual skull and the dura mater were removed with a forceps clip. The left middle cerebral artery (L- 
MCA) was exposed, and the L-MCA was cleaved by a bipolar coagulator at the proximal olfactory bundle. The muscle tissues and salivary glands were reset, and antibiotics were added to the surgical area, wiped with $0.9 \%$ sodium chloride solution and sutured. The experimental animals were placed in an incubator after the surgery, and the relevant experiments were performed after the animals were awake. The body temperature of the rats was controlled at $36.5-37^{\circ} \mathrm{C}$ during the surgery. In total, 20 rats were successfully operated upon. The sham-operated rats were treated as above but without the electrocoagulation. All the rats survived after surgery. Five of them died within 2 weeks, and 15 rats were successfully modeled. The success rate of modeling reached $75 \%$ at 2 weeks. During grouping, there were 5 normal rats, 5 shamoperated rats, 5 MCAO rats, 5 MCAO rats stimulated with $1 \mathrm{~Hz}$ rTMS and 5 MCAO rats stimulated with 10 $\mathrm{Hz}$ rTMS. rTMS was started 3 days after MCAO-induced ischemic injury [19] as shown in Supplementary Fig. 1.

\section{Neurobehavioral evaluation of rats}

Neurological evaluation was performed on days 1, 3, 5, 7 and 14 following MCAO-induced ischemic injury. The rTMS instrument was produced by Wuhan Yiruide Medical Equipment Co., Ltd. (Wuhan, Hubei, China), with a maximum stimulation frequency of $100 \mathrm{~Hz}$ and a circular stimulation coil with diameter of $6 \mathrm{~cm}$. The stimulation intensity was $70 \%$ of the maximum output intensity. Primary neurons were placed under a transcranial magnetic stimulator and given low $(1 \mathrm{~Hz}$ for $900 \mathrm{~s})$ and high ( $10 \mathrm{~Hz}$ for $10 \mathrm{~s}$ at an interval of $50 \mathrm{~s}$, total number of pluses of 900 ) frequency stimulation. The rats used for control were also placed under the stimulation device but without stimulation. After the end of the treatment, the cells were further incubated for $3 \mathrm{~h}$ for subsequent experiments. After inducing for $24 \mathrm{~h}$, the rats in the rTMS groups were treated with rTMS once a day for 2 weeks at low $(1 \mathrm{~Hz}$ for $900 \mathrm{~s})$ and high frequency $(10 \mathrm{~Hz}$ for $10 \mathrm{~s}$ at an interval of $50 \mathrm{~s}$, total number of pluses was 900 ) conditions. All the rats were given free access to food and water during the experiment.The neurological evaluation of rats was performed by two informed observers based on the following standards: (1) score with the reference to the Bederson neurological deficit, with the score range of 0 - 3; the higher score indicated the more severe neurological deficit; (2) score of the wire gripping ability with the score range of 0 - 3; (3) learning and memory ability test: the rats were tested for learning and memory ability by $\mathrm{Y}$-type electrical maze after rTMS treatment, and the number of correct responses in each group was recorded. Each group of rats was tested 3 times

for averaging. On the $14^{\text {th }} \mathrm{d}$ after injury, all rats were injected with $2 \%$ pentobarbital sodium and ketorolac tromethamine to relieve pain. The left ventricle was perfused with $200 \mathrm{~mL} 0.9 \%$ sodium chloride solution. The perfused rats were immediately euthanized with $\mathrm{CO}_{2}$ inhalation, and the brain was extracted and stored at $-70^{\circ} \mathrm{C}$ for future use.

\section{Reverse transcription-quantitative polymerase chain reaction (RT-qPCR)}


A total of $1 \mathrm{~mL}$ Trizol reagent (Invitrogen, Carlsbad, CA, USA) was added to samples while they were simultaneously pulverized in an ice bath. Total RNA was extracted using an RNA extraction kit (Qiagen company, Hilden, Germany) for real-time PCR. The RNA was reverse transcribed into cDNA $(50 \mathrm{ng} / \mu \mathrm{L})$ using the PrimeScript ${ }^{\mathrm{TM}}$ RT reagent Kit (RR047A, Beijing Zhijie Fangyuan Technology Co., Ltd., Beijing, China). Primers (Table 1) were designed using primer Premier 5.0 software and synthesized by Beijing Qingke Biotechnology Co., Ltd. (Beijing, China). RT-qPCR was performed on the ABI 7900HT real-time quantitative PCR instrument (ABI 7900, Shanghai Pudi Biotechnology Co., Ltd., Shanghai, China) using a two-step method, with $\beta$-actin as an internal reference. The TrkB mRNA expression was determined by the $2^{-\Delta \Delta C t}$ method. Three replicate wells were set for each gene of each sample. The experiment was repeated three times.

\section{Western blot analysis}

The total protein in the cerebral cortical tissues was extracted with Radio-Immunoprecipitation assay (RIPA) cell lysis buffer containing phenylmethanesulfonyl fluoride (PMSF) (R0010, Beijing Solarbio Life Sciences Co., Ltd., Beijing, China), incubated on ice for $30 \mathrm{~min}$, and centrifuged at $12000 \times \mathrm{g}$ for $10 \mathrm{~min}$ at $4^{\circ} \mathrm{C}$. The supernatant was extracted for protein quantitative detection. An amount of $50 \mu \mathrm{g}$ protein sample was dissolved in $2 \times$ sodium dodecyl sulfate (SDS) loading buffer, and boiled at $100^{\circ} \mathrm{C}$ for 5 min. Subsequently, the proteins were separated using 10\% SDS-polyacrylamide gel electrophoresis (PAGE) at $200 \mathrm{~V}$ for $50 \mathrm{~min}$, and the electrophoresis was terminated once the bromophenol blue reached the bottom of the separation gel. Then, the protein sample was transferred to a polyvinylidene fluoride (PVDF) membrane and blocked with $5 \%$ skim milk at room temperature for $1 \mathrm{~h}$, and rinsed with phosphate buffered saline (PBS) for $2 \mathrm{~min}$. The membrane was incubated with diluted rabbit polyclonal antibodies specific to glyceraldehyde-3-phosphate dehydrogenase (GAPDH; ab9485), TrkB (ab18987), p-TrkB (ab228507), p-CaMKIV (ab195000), CaMKIV (\#4032), CREB (\#9197), p-CREB (\#9198), PKA (\#4782) or pPKA (\#5661) at $4^{\circ} \mathrm{C}$ overnight. Antibodies to GAPDH, TrkB, p-TrkB and p-CaMKIV were obtained from Abcam Inc. (Cambridge, UK), and antibodies to CaMKIV, CREB, p-CREB, PKA and p-PKA were obtained from Cell Signaling Technology (Beverly, MA, USA). After overnight incubation, the membrane was washed three times with Tris buffered saline tween-20 (TBST; $5 \mathrm{~min}$ ) prior to incubation with horse reddish peroxidase (HRP)-labeled goat anti-rabbit immunoglobulin G (IgG; $1: 1000$, HA1003, Shanghai Yanhui Biotechnology Co., Ltd., Shanghai, China) for $1 \mathrm{~h}$. The membrane was reacted with electrogenerated chemiluminescence (ECL) solution (ECL808-25, Biomiga, San Diego, CA, USA) at room temperature for $1 \mathrm{~min}$ and visualized.

\section{Chromatin immunoprecipitation (ChIP)}

The cerebral cortical tissue was cut into 2-mm pieces, cross-linked in $1 \%$ formaldehyde and stored at $-80^{\circ} \mathrm{C}$ for later use. The nucleus was extracted with microtip, lysed, and sonicated until the DNA fragment 
was about $200-1000$ bp in length. Next, $40 \mu \mathrm{L}$ Protein $\mathrm{G}$ beads and $3 \mu \mathrm{g}$ antibody were pre-incubated together for at least $1 \mathrm{~h}$ prior to overnight incubation with the DNA fragment for immunoprecipitation. The DNA/protein complex was eluted from the beads and reversely cross-linked overnight at $65^{\circ} \mathrm{C}$. The DNA was detached by ribonuclease $\mathrm{A}$ and proteinase $\mathrm{K}$, followed by phenol/chloroform extraction and alcohol precipitation. Real-time quantitative PCR was performed using iQ SYBR Green Supermix (Bio-Rad Laboratories, Shanghai, China). The amount of immunoprecipitated DNA was calculated by comparison with the total amount of input DNA. Data obtained represent four independent amplifications. ChIP was carried out with CREB antibody, and GPCR quantified the exon of the precipitated TrkB at CRE sites.

\section{Fractionation of cytoplasmic and nuclear proteins}

Cytoplasmic and nuclear fractions were isolated according to the instructions of the Nuclear Protein Extraction Kit (C500009-0050, Shanghai Sangon Biotech Co., Ltd., Shanghai, China). The internal references of nuclear and cytoplasmic proteins were lamin A (ab26300) and GAPDH (ab9485), respectively.

\section{Co-Immunoprecipitation}

The cells or tissues were collected for co-immunoprecipitation. A part of the fragmented chromatin was taken as an input before immunoprecipitation. The cells or tissues were precipitated with polyclonal antibody specific to TrkB (ab18987, 1 : 1000, Abcam Inc., Cambridge, MA, USA), washed two times with pre-cooled PBS, and fully lysed. After centrifuged at $25764 \times \mathrm{g}$ for $3-5 \mathrm{~min}$, the supernatant was collected and probed with primary antibody at $4^{\circ} \mathrm{C}$ overnight. Then, $50 \%$ agarose beads at a volume ratio of $\mathrm{I}: 10$ were added and fully mixed at $4^{\circ} \mathrm{C}$ for $10 \mathrm{~min}$. After centrifugation for $15 \mathrm{~min}$ at $118 \times \mathrm{g}$ and $4^{\circ} \mathrm{C}$, the supernatant was discarded and the agarose beads remained. After the last wash, SDS protein loading buffer was added to the cells or tissues and then they were mixed, boiled for $5 \mathrm{~min}$, and centrifuged at $25764 \times \mathrm{g}$ for $3 \mathrm{~min}$. The supernatant was then collected for SDS-PAGE electrophoresis analysis. Primary antibodies (Abcam Inc., Cambridge, UK) to phospholipase C gamma 1 (PLC-y1; ab76155, 1 : 5000), shc (ab33770, $1: 1000$ ) and N-methyl-D-aspartate receptor subunit NR1 (NMDAR-NR1; ab134308, $1: 1000$ ) were used for Western blot analysis. The non-specific rat IgG at $1: 50$ was added as NC. The above experiments were repeated 3 times.

\section{Detection of intracellular $\mathrm{Ca}^{2+}$ concentration}

$\mathrm{Ca}^{+}$fluorescent probe Fura-2/AM was mixed with the calcium-containing solution at a ratio of 1:499, and then added into each group of cells. The cells were then incubated at $37^{\circ} \mathrm{C}$ and de-esterified. The fluorescence of Fura-2/AM was excited using excitation light at wavelengths of 340 and $380 \mathrm{~nm}$. The 
dynamic change of fluorescence was monitored by Charge coupled device (CCD) and analyzed by the calcium fluorescence imaging system IPA software. The change value of the fluorescence intensity ratio of the two excitation lights ( $\Delta$ ratio) reflected the intracellular calcium ion concentration $\left[\mathrm{Ca}^{2+}\right]$ i.

\section{Enzyme-linked immunoassay (ELISA)}

A total of $100 \mathrm{pL}$ enzyme immunoassay (EIA) buffer was added to the non-specific binding wells, and 50 $\mu \mathrm{L}$ EIA Buffer was added to the largest amount of binding well. Next, $50 \mu \mathrm{L}$ buffer was pipetted from the No. 8 tube into the two standard wells of the lowest sections, and another $50 \mu \mathrm{L}$ buffer was pipetted from the No. 7 tube into the next two standard wells. This procedure was continued until all standards were added into the standard wells. In addition to the total activity (TA) and blank wells, $50 \mu \mathrm{L}$ cAMP acetylcholinesterase tracer was added to each well. In addition to TA, nonspecific binding (NSB), and blank wells, $50 \mu \mathrm{L}$ cAMP EIA antiserum was added to each well. The plate was covered with plastic film and incubated at $4^{\circ} \mathrm{C}$ for $18 \mathrm{~h}$. The liquid in the wells was discarded, and the wells were washed five times with wash buffer, followed by the addition of $200 \mu \mathrm{L}$ Ellman reagent into each well. Afterwards, $5 \mathrm{pL}$ of tracer agent was added into the TA well. The plate was then covered with plastic film, wrapped with tin foil paper, and shaken for $2 \mathrm{~h}$ on the shaker. The multi-function microplate reader was used to read the optical density (OD) value at $412 \mathrm{~nm}$.

\section{Triphenyltetrazolium chloride (TTC) staining}

The fresh brain tissues were placed in a refrigerator at $-20^{\circ} \mathrm{C}$ for $10 \mathrm{~min}$, and sliced into $1-\mathrm{mm}$ sections. The sections were incubated with PBS containing $1 \% \mathrm{TTC}(\mathrm{pH}=7.4)$ in a $37^{\circ} \mathrm{C}$ water bath for $20 \mathrm{~min}$. The staining solution was shaken to ensure adequate contact with brain tissue. After the staining was terminated, the sections were fixed in $10 \%$ formaldehyde overnight. The viable brain tissue was stained in brick red color, and the infarct area in grayish white color. The ImagemasterVDS image analyzer was used to analyze the percentage of infarct area in the brain tissues to the total brain tissue. Cerebral infarct area ratio $(\%)=$ sum of infarct area of all sections/total area of brain $\times 100 \%$.

\section{Immunofluorescence}

Cortex, hippocampus and striatum were extracted after the experimental animals were fixed, perfused and dehydrated. Afterwards, $30 \mu \mathrm{M}$-thick brain sections were made using a freezing microtome. After non-specific blocking, the sections were incubated in antibody solution containing anti-rat synaptophysin (Millipore, Billerica, MA, USA) or anti-rabbit PSD-95 (Millipore, Billerica, MA, USA) at $4^{\circ} \mathrm{C}$ for $24 \mathrm{~h}$. After being washed with PBS, the sections were incubated with anti-Alexa-488 and 568-labeled secondary antibodies, respectively. The sections were then placed under a Nikon fluorescence microscope (TE2000, 
Nikon, Tokyo, Japan) to observe the staining intensity. The average fluorescence intensity was determined by the ImageJ software package. Immunohistochemical stainingThe rat cortex was fixed in $10 \%$ neutral formalin solution, dehydrated for $24 \mathrm{~h}$ with gradient ethanol, embedded in paraffin for $12 \mathrm{~h}$, and cut into 5 slices with a thickness of about $3-4 \mu \mathrm{m}$. The sections were dewaxed with xylene I and II for 10 min respectively, dehydrated by gradient ethanol (100\%, 95\%, $80 \%$ and $70 \%$ for 2 min respectively), and then washed twice with PBS ( 5 min for each time). The sections were then soaked in $3 \% \mathrm{H}_{2} \mathrm{O}_{2}$ for 10 min and washed twice with PBS (5 min for each time). After antigen retrieval under high-pressure for $90 \mathrm{~s}$, the sections were cooled at room temperature and incubated with $5 \% \mathrm{BSA}$ blocking solution at $37^{\circ} \mathrm{C}$ for $30 \mathrm{~min}$. Subsequently, the sections were incubated with $50 \mu \mathrm{L}$ rabbit anti-mouse antibody to CREB (ab32515; $1: 100$ ) at $4^{\circ} \mathrm{C}$ overnight, followed by incubation with $50 \mu \mathrm{L}$ biotinylated mouse anti-goat IgG (SF8-0.3, 1 : 100, Beijing Solarbio Life Sciences Co., Ltd., Beijing, China) at $37^{\circ} \mathrm{C}$ for $30 \mathrm{~min}$. Streptavidinbiotin-peroxidase (SAB) working solution was added to the sections prior to development with diaminobenzidine (DAB) and counterstaining with hematoxylin for $5 \mathrm{~min}$, followed by dehydration, clear, sealing and microscopic examination. The primary antibody was replaced by PBS buffer for the NC. Four high power visual fields $(\times 400)$ were randomly selected for each section (200 cells per visual field). The criteria for CREB protein positive cells were that the normal positive cells were brownish yellow and; the positive expression rate $=$ the number of cells with positive expression/the total number of cells $\times 100 \%$. The experiment was repeated 3 times.

\section{Statistical analysis}

All data were statistically analyzed using a Statistic Package for Social Science (SPSS) 21.0 (IBM Corp. Armonk, NY, USA). The measurement data were expressed as mean \pm standard deviation. The data of multiple groups obeying the normal distribution and homogeneity of variance were compared using oneway analysis of variance (ANOVA) with Tukey's post-hoc test, and data at different time points were compared using repeated measurement analysis of variance. The difference was statistically significant at $p<0.05$.

\section{Results}

rTMS enhanced the nerve recovery ability of MCAO rats and increased TrkB expression

The rat model with cerebral ischemia was developed using MCAO. Neurobehavioral evaluation at days 1 , 3,7 and 14 after injury and NeuN immunofluorescence staining were conducted to confirm the successful establishment of the rat model. As demonstrated in Table 2, MCAO rats were weaker than normal and sham-operated rats in terms of neurological function, motion balance, and learning and memory function 
$(p<0.05)$. It was found that the fluorescence intensities of NeuN in the cortex, hippocampus and striatum of MCAO rats were significantly lower than that in the normal and sham-operated rats $(p<0.05$, Fig. $1 \mathrm{~A})$. The above results suggested that the rat model of permanent focal cerebral ischemia was successfully established, which was characterized by most obvious cortical changes.

In order to evaluate whether rTMS stimulation could improve the neurological function of MCAO rats, the neurobehavioral changes in rats were examined at days 1, 3, 7 and 14 after injury. The results showed that both $1 \mathrm{~Hz}$ and $10 \mathrm{~Hz}$ rTMS increased the neurological function, motion balance, learning and memory function of MCAO rats (Table 3). NeuN immunofluorescence of cortex demonstrated that the fluorescence intensity of NeuN in the MCAO rats was significantly increased following the stimulation of $1 \mathrm{~Hz}$ or $10 \mathrm{~Hz}$ rTMS (Fig. 1B). The infarct volume in brain tissue was detected by TTC staining, which showed that the infarct volume of brain tissue was decreased in the MCAO rats after stimulation with 1 $\mathrm{Hz}$ or $10 \mathrm{~Hz}$ rTMS (Fig. 1C). The immunohistochemical detection of TrkB revealed that after MCAO model establishment, either $1 \mathrm{~Hz}$ or $10 \mathrm{~Hz}$ rTMS increased the positive expression of TrkB in MCAO rats (Fig. 1D). RT-qPCR and Western blot analysis illustrated that the expression of TrkB was upregulated and the TrkB phosphorylation in the MCAO rats was enhanced following stimulation with $1 \mathrm{~Hz}$ or $10 \mathrm{~Hz}$ rTMS (Fig. 1E-F). The above results demonstrated that rTMS could enhance the neurological recovery and increase the expression of TrkB in rats with cerebral ischemia.

\section{rTMS activates CREB via the CAMP/PKA pathway}

The primary neurons of the cerebral cortex were treated with OGD and placed under a transcranial magnetic stimulator to receive low $(1 \mathrm{~Hz})$ and high frequency $(10 \mathrm{~Hz})$ stimulation. The protein expression of CREB and PKA and their phosphorylation levels were measured by Western blot analysis. As depicted in Fig. 2A and B, compared with the OGD-treated neurons, the expression of CREB and PKA were unchanged, and their phosphorylation levels were significantly up-regulated in the OGD-treated neurons after stimulation with $1 \mathrm{~Hz}$ or $10 \mathrm{~Hz}$ rTMS. The results of ELISA showed that the CAMP expression was increased by stimulation with $1 \mathrm{~Hz}$ or $10 \mathrm{~Hz}$ rTMS in the OGD-treated neurons (Fig. 2C). The results of immunofluorescence in Fig. 2D illustrated that compared with the OGD-treated neurons without stimulation, the fluorescence intensity of PKA was significantly increased in the nucleus of the OGDtreated neurons stimulated with $1 \mathrm{~Hz}$ or $10 \mathrm{~Hz}$, but significantly decreased in the cytoplasm $(p<0.05)$. After nuclear and cytoplasmic protein separation, the expression of PKA, GAPDH, and Lamin A protein in the nucleus and cytoplasm were analyzed by Western blot analysis. The results revealed that the OGDtreated neurons had increased PKA protein expression in the nucleus but decreased PKA protein expression in the cytoplasm after stimulation of $1 \mathrm{~Hz}$ or $10 \mathrm{~Hz}$ rTMS (Fig. 2E). According to the coimmunoprecipitation and Western blot analysis, compared with the OGD-treated neurons without stimulation, PKA antibody in the OGD-treated neurons stimulated with $1 \mathrm{~Hz}$ or $10 \mathrm{~Hz}$ bound to more CREB $(p<0.05$, Fig. 2F). When the expression of PKA was down-regulated, the expression of CREB and its phosphorylation were determined by Western blot analysis. The results showed that after $1 \mathrm{~Hz}$ or $10 \mathrm{~Hz}$ rTMs, the expression of CREB was unchanged, while its phosphorylation was significantly decreased in the OGD-treated neurons by the infection of adenovirus expressing siPKA (Fig. 2G). These results 
suggested that rTMS increased the expression of CAMP, CREB and PKA phosphorylation in primary cultured cortical neurons, stimulated the translocation of PKA into nuclei, as well as enhanced the interaction between PKA and CREB, while siPKA could inhibit the extent of CREB phosphorylation. As described above, rTMS activated CREB via the activation of the CAMP/PKA pathway.

\section{rTMS activates CREB via activating the $\mathrm{Ca}^{2+} /$ CaMKIV pathway}

rTMS has been demonstrated to increase the extent of CREB phosphorylation. OGD treatment was also performed on primary cortical neurons which were then placed under the transcranial magnetic stimulator, respectively with low $(1 \mathrm{~Hz})$ and high frequency $(10 \mathrm{~Hz})$ stimulation. The fluorescent probe Fura- 2 was used to determine intracellular $\mathrm{Ca}^{2+}$ concentration. The results showed (Fig. 3A) that when compared with OGD-treated neurons without stimulation, [Ca2+]i was significantly higher in OGD-treated neurons stimulated with $1 \mathrm{~Hz}$ or $10 \mathrm{~Hz}$ rTMS $(p<0.05)$. Western blot analysis showed that the expression of CaMKIV was unchanged, and its phosphorylation was significantly higher in OGD-treated neurons stimulated with $1 \mathrm{~Hz}$ or $10 \mathrm{~Hz}$ rTMS when compared with OGD-treated neurons without stimulation ( $p<$ 0.05; Fig. 3B). After CaMKIV expression was down-regulated, the expression of CREB and its phosphorylation were measured by Western blot analysis. As shown in Fig. 3C, siCaMKIV inhibited CREB phosphorylation in OGD neurons treated by $1 \mathrm{~Hz}$ or $10 \mathrm{~Hz}$ rTMS. These results indicated that $1 \mathrm{~Hz}$ or 10 $\mathrm{Hz}$ rTMS potentiated CREB phosphorylation by activating the Ca2+/CaMKIV pathway.

\section{rTMS upregulates TrkB by activating CREB}

The primary neurons of cerebral cortex were treated with OGD and placed under a transcranial magnetic stimulator, and given low $(1 \mathrm{~Hz})$ and high frequency $(10 \mathrm{~Hz})$ stimulation respectively. The results of RTqPCR of TrkB mRNA expression showed that, when compared with the OGD-treated neurons without stimulation, the mRNA expression of TrkB was significantly higher in the OGD-treated neurons stimulated with $1 \mathrm{~Hz}$ or $10 \mathrm{~Hz}$ rTMS ( $p<0.05$; Fig. 4A). The protein expression of TrkB and its phosphorylation determined by Western blot analysis revealed that OGD neurons had unchanged expression of TrkB but enhanced TrkB phosphorylation with the stimulation of $1 \mathrm{~Hz}$ or $10 \mathrm{~Hz}$ rTMS (Fig. 4B). The binding of CREB to TrkB at CRE sites in ChIP assay is shown in Fig. 4C. Compared with OGD-treated neurons without stimulation, enrichment of TrkB at CRE sites in OGD-treated neurons stimulated with $1 \mathrm{~Hz}$ or $10 \mathrm{~Hz} \mathrm{rTMS}$ was significantly higher $(p<0.05)$. After CREB was down-regulated, the RT-qPCR determination of TrkB mRNA expression showed that the treatment of siCREB decreased the mRNA expression of TrkB in OGDtreated neurons stimulated with $1 \mathrm{~Hz}$ or $10 \mathrm{~Hz}$ rTMS (Fig. 4D). Western blot analysis was used to determine the expression of TrkB and its phosphorylation (Fig. 4E), and the results showed that siCREB decreased the extent of TrkB phosphorylation in OGD neurons induced by $1 \mathrm{~Hz}$ or $10 \mathrm{~Hz}$ rTMS with unchanged expression of TrkB. Co-immunoprecipitation and Western blot analysis were employed to characterize the expression of PLC- $y 1$, shc and NMADR-NR1 co-immunoprecipitated with TrkB (Fig. 4F). The results demonstrated that when compared with OGD-treated neurons, the expression of PLC- $\gamma 1$, shc and NMADR-NR1 co-immunoprecipitated with TrkB was significantly increased in OGD-treated neurons stimulated with $1 \mathrm{~Hz}$ or $10 \mathrm{~Hz}$ rTMS $(p<0.05)$. After CREB was down-regulated, the expression of PLC- $y 1$, 
SHC and NMADR-NR1 co-immunoprecipitated with TrkB was significantly decreased in OGD-treated neurons stimulated with $1 \mathrm{~Hz}$ or $10 \mathrm{~Hz}$ rTMS (Fig. 4G). These results indicate that rTMS could increase the TrkB expression, enhance the binding of CREB to TrkB at CRE sites, and promote the recruitment ability of TrkB, while siCREB could inhibit the TrkB expression. In summary, rTMS increased the TrkB expression by activating CREB.

\section{rTMS promotes the binding of CREB to TrkB in vivo}

The effect of rTMS on the interaction between CREB and TrkB was assessed in MCAO rats. The protein expression of CREB and its phosphorylation were determined by Western blot analysis (Fig. 5A). When compared with the MCAO rats, the expression of CREB in MCAO rats stimulated with $1 \mathrm{~Hz}$ or $10 \mathrm{~Hz}$ rTMS was unchanged, and the extent of CREB phosphorylation was significantly increased $(p<0.05)$. The results of immunohistochemical detection, the positive rate of phosphorylated CREB protein in the MCAO rats stimulated with $1 \mathrm{~Hz}$ or $10 \mathrm{~Hz}$ rTMS was significantly higher than that in the MCAO rats without stimulation ( $p<0.05$; Fig. 5B). The cAMP expression measured by ELISA showed that $1 \mathrm{~Hz}$ or $10 \mathrm{~Hz}$ rTMS both increased the CAMP expression in the MCAO rats (Fig. $5 \mathrm{C}$ ). Compared with the MCAO rats, the expression of PKA in the MCAO rats stimulated with $1 \mathrm{~Hz}$ or $10 \mathrm{~Hz}$ rTMS was unchanged, while the extent of its phosphorylation was notably increased $(p<0.05$; Fig. 5D). The expression of CaMKIV and its phosphorylation showed changes consistent with PKA ( $p<0.05$; Fig. 5E). The co-immunoprecipitation and Western blot analysis demonstrated that the amount of TrkB co-immunoprecipitated with CREB was significantly higher in the MCAO rats stimulated with $1 \mathrm{~Hz}$ or $10 \mathrm{~Hz}$ rTMS than in the MCAO rats without stimulation $(p<0.05$; Fig. $5 \mathrm{~F}$ ). The above results suggested that rTMS could increase the expression of CAMP and enhance CREB, PKA and CaMKIV phosphorylation, hence promoting the binding of CREB to TrkB in vivo.

\section{Discussion}

rTMS has been reported to regulate progression in the human brain, and was associated with blood flow and oxygenation changes in the cerebral cortex, suggesting its potential role as a therapeutic technique for neurologic conditions [20]. Therefore, this study investigated the specific roles of rTMS in neural function after cerebral ischemia in rats. Collectively, the present study has demonstrated that rTMS could improve neurological function after cerebral ischemic in rats by increasing the expression of TrkB via activation of the CREB.

By establishing the OGD neuron model and the MCAO rat model, this study discovered that rTMS could enhance the nerve recovery ability of rats with cerebral ischemia, accompanied by high expression of TrkB. rTMS functions as a nonsurgical technique for cerebral stimulation with promising effects and causes changes in neuroplasticity, thus holding therapeutic potential for neurological diseases [21]. rTMS over motor cortex could promote recovery, which may be beneficial in acute stroke therapy [22]. In addition, rTMS could exert its function on the reduction of unilateral spatial neglect and improve upper extremity function after subacute stroke, subsequently stimulating neuron depolarization and changing 
the excitability within the cerebral cortex after rMS [23]. rTMS with low intensity elicits the activation of BDNF and TrkB, which further inhibits the dysfunction of spatial cognition in aging mice [24]. A smallmolecule TrkB agonist 7,8-dihydroxyflavone has been reported to alleviate cerebral ischemia and reperfusion injury in rats [25]. Hence, the neurological function of rats with cerebral ischemia could be improved following treatment with rTMS and upregulation of TrkB, which was consistent with the results of this study.

In addition, our study demonstrated that rTMS could activate CREB through the CAMP/PKA and $\mathrm{Ca}^{2+} /$ CaMKIV pathways by elevating $\left[\mathrm{Ca}^{2+}\right] \mathrm{i}$, and the extent of CREB, CAMP, PKA and CaMKIV phosphorylation. CAMP is an intracellular second messenger that exerts various physiological effects through activation of PKA where the CAMP/PKA pathway is associated with neural control of glucose homeostasis in mammals [26]. The cAMP/PKA pathway has been reported to play a significant role in the transcriptional activities of CREB [27]. Based on the data on neuronal differentiation from a study by Hellmann et al.,, rTMS promotes the formation of CAMP and the consequent phosphorylation of CREB [8]. $\mathrm{Ca}^{2+} / \mathrm{CaMKK}$, a novel element of the caMK family, could induce the activation and phosphorylation of specific downstream protein kinases to trigger various $\mathrm{Ca}^{2+}$-signaling pathways, which is vital for modulation of gene expression regulated by CREB phosphorylation [28]. The $\mathrm{Ca}^{2+} / \mathrm{CaMKIV}$ pathway has been linked to the activation of transcription factors such as CREB [29]. In addition, $\mathrm{Ca}^{2+}$-dependent $\mathrm{CREB} / \mathrm{c}$-fos activation by $\mathrm{Ca}^{2+}{ }^{2+} \mathrm{CaMKIV}$ triggers osteoclast-specific gene regulation at transcriptional level through NFATCl [30]. Moreover, the high-frequency rTMS caused CREB phosphorylation and transcription of BDNF through the activation of the $\mathrm{Ca}^{2+}$-CaMKII-CREB pathway in the Neuro-2a cells [31]. As a member of the CaMK family, $\mathrm{Ca}^{2+} / \mathrm{CaMKIV}$ along with its protein product was significantly detected in the nucleus, which modulates gene transcription by CREB phosphorylation and activation [32]. From the above reported studies, it could be concluded that rTMS induced the activation of CREB via the cAMP/PKA and $\mathrm{Ca}^{2+} /$ CaMKIV pathways.

Another significant finding in this study was that rTMS could increase the TrkB expression through activating CREB. A previous study has demonstrated that magnetic stimulation with low frequency could modulate structural synaptic plasticity of hippocampal neurons by activating BDNF-TrkB pathways [13]. As a member of the neurotrophin family, BDNF improves the functional recovery and triggers neuroprotective effects after ischemic stroke, and its protective effects are exerted by binding to the highaffinity TrkB receptor [33]. It has also been demonstrated that daily $5 \mathrm{~Hz}$ rTMS for $5 \mathrm{~d}$ enhanced the BDNF-TrkB pathway in both cortex and lymphocytes by elevating the demand of BDNF for TrkB [34]. Also, the CREB activation could result in increased expression of anti-apoptotic protein $\mathrm{Bcl}-2$, and is conducive to neuronal survival after ischemic attack [35]. Overall, the aforementioned findings are supportive of our results where rTMS activated CREB and TrkB, thereby improving neurological function under cerebral ischemic conditions.

\section{Conclusions}


In brief, rTMS could contribute to the protection of patients against cerebral ischemia by improving the neurological function via activating CREB, to regulate TrkB through the CAMP/PKA and $\mathrm{Ca}^{2+} / \mathrm{CaMKIV}^{2}$ pathways (Fig. 6). This finding suggests that rTMS is a promising therapeutic module for patients who have suffered from cerebral ischemia. Nevertheless, in-depth investigations on the function of rTMS in cerebral ischemia are ongoing and further exploration of the underlying mechanisms of rTMS is necessary. Despite showing promise for prognostic and therapeutic treatments in cerebral ischemia, clinical experiments of fully developed rTMS-based therapeutic treatments are needed in the future.

\section{Abbreviations}

rTMS: Repetitive transcranial magnetic stimulation; OGD: oxygen-glucose deprivation; MCAO: middle cerebral artery occlusion; CREB: cAMP-response element binding protein; BDNF: brain-derived neurotrophic factor; TrkB: tropomyosin-related kinase B; SD: Sprague Dawley; SPF: specific pathogen free; WT: wild type; siNC: siRNA negative control; siCREB: siRNA targeting CREB; siPKA: siRNA targeting PKA; siCaMKIV: siRNA targeting CaMKIV; DMEM: Dulbecco's modified eagle medium; BSA: bovine serum albumin; OGD: oxygen-glucose deprivation; MCAO: middle cerebral artery occlusion; L-MCA: left middle cerebral artery; RT-qPCR: Reverse transcription-quantitative polymerase chain reaction; RIPA: RadioImmunoprecipitation assay; PMSF: phenylmethanesulfonyl fluoride; SDS: sodium dodecyl sulfate; PAGE: polyacrylamide gel electrophoresis; PVDF: polyvinylidene fluoride; PBS: phosphate buffered saline; HRP: horse reddish peroxidase; ECL: electrogenerated chemiluminescence; ChIP: Chromatin immunoprecipitation; ELISA: Enzyme-linked immunoassay; EIA: enzyme immunoassay; TA: total activity; NSB: nonspecific binding; OD: optical density; TTC: Triphenyltetrazolium chloride; SAB: Streptavidinbiotin-peroxidase.

\section{Declarations}

\section{Acknowledgements}

We would like to give our sincere appreciation to the reviewers for their helpful comments on this article.

\section{Authors' contributions}

Lisha Chang, Zhaowang An, and Fuling Zhou designed the study. Dali Wang, and Jian Liu collated the data, Yunhe Zhang designed and developed the database, Jiang Zhang carried out data analyses and produced the initial draft of the manuscript. Lisha Chang and Zhaowang An contributed to drafting the manuscript. All authors have read and approved the final submitted manuscript.

\section{Funding}


None.

\section{Availability of data and materials}

All data in this article is available.

\section{Ethics approval and consent to participate}

The animal experiments were performed in strict accordance with the recommendations in the Guide for the Care and Use of Laboratory Animals of the National Institutes of Health. The protocol was approved by the Animal Ethics Committee of North China University of Science and Technology Affiliated Hospital.

\section{Consent for publication}

Not applicable.

\section{Competing interests}

The authors declare that they have no competing interests.

\section{Author details}

1 The 2nd Department of Neurology, North China University of Science and Technology Affiliated Hospital, Tangshan 063000, P.R. China. ${ }^{2}$ Department of Neurosurgery, North China University of Science and Technology Affiliated Hospital, Tangshan 063000, P.R. China

\section{References}

1. Radak D, Resanovic I, Isenovic ER. Link between oxidative stress and acute brain ischemia. Angiology. 2014;65:667-76. 
2. Wang F, Liang W, Lei C, Kinden R, Sang H, Xie Y, Huang Y, Qu Y, Xiong L. Combination of HBO and Memantine in Focal Cerebral Ischemia: Is There a Synergistic Effect? Mol Neurobiol. 2015;52:145866.

3. Li P, Gan Y, Sun BL, Zhang F, Lu B, Gao Y, Liang W, Thomson AW, Chen J, Hu X. Adoptive regulatory Tcell therapy protects against cerebral ischemia. Ann Neurol. 2013;74:458-71.

4. Ishii T, Asai T, Oyama D, Agato Y, Yasuda N, Fukuta T, Shimizu K, Minamino T, Oku N. Treatment of cerebral ischemia-reperfusion injury with PEGylated liposomes encapsulating FK506. FASEB J. 2013;27:1362-70.

5. Lan L, Tao J, Chen A, Xie G, Huang J, Lin J, Peng J, Chen L. Electroacupuncture exerts antiinflammatory effects in cerebral ischemia-reperfusion injured rats via suppression of the TLR4/NFkappaB pathway. Int J Mol Med. 2013;31:75-80.

6. Yoon KJ, Lee YT, Han TR. Mechanism of functional recovery after repetitive transcranial magnetic stimulation (rTMS) in the subacute cerebral ischemic rat model: neural plasticity or anti-apoptosis? Exp Brain Res. 2011;214:549-56.

7. Beom J, Kim W, Han TR, Seo KS, Oh BM. Concurrent use of granulocyte-colony stimulating factor with repetitive transcranial magnetic stimulation did not enhance recovery of function in the early subacute stroke in rats. Neurol Sci. 2015;36:771-7.

8. Hellmann J, Juttner R, Roth C, Bajbouj M, Kirste I, Heuser I, Gertz K, Endres M, Kronenberg G. Repetitive magnetic stimulation of human-derived neuron-like cells activates cAMP-CREB pathway. Eur Arch Psychiatry Clin Neurosci. 2012;262:87-91.

9. Benito E, Barco A. CREB's control of intrinsic and synaptic plasticity: implications for CREBdependent memory models. Trends Neurosci. 2010;33:230-40.

10. Zhang L, Zhao H, Zhang $X$, Chen L, Zhao X, Bai X, Zhang J. Nobiletin protects against cerebral ischemia via activating the $\mathrm{p}-\mathrm{Akt}, \mathrm{p}-\mathrm{CREB}, \mathrm{BDNF}$ and $\mathrm{Bcl}-2$ pathway and ameliorating $\mathrm{BBB}$ permeability in rat. Brain Res Bull. 2013;96:45-53.

11. Li N, Liu GT. The novel squamosamide derivative FLZ enhances BDNF/TrkB/CREB signaling and inhibits neuronal apoptosis in APP/PS1 mice. Acta Pharmacol Sin. 2010;31:265-72.

12. Odate S, Nakamura K, Onishi H, Kojima M, Uchiyama A, Nakano K, Kato M, Tanaka M, Katano M. TrkB/BDNF signaling pathway is a potential therapeutic target for pulmonary large cell neuroendocrine carcinoma. Lung Cancer. 2013;79:205-14.

13. Ma J, Zhang Z, Su Y, Kang L, Geng D, Wang Y, Luan F, Wang M, Cui H. Magnetic stimulation modulates structural synaptic plasticity and regulates BDNF-TrkB signal pathway in cultured hippocampal neurons. Neurochem Int. 2013;62:84-91.

14. Yao RQ, Qi DS, Yu HL, Liu J, Yang LH, Wu XX. Quercetin attenuates cell apoptosis in focal cerebral ischemia rat brain via activation of BDNF-TrkB-PI3K/Akt signaling pathway. Neurochem Res. 2012;37:2777-86.

15. Yang J, Shi QD, Song TB, Feng GF, Zang WJ, Zong CH, Chang L. Vasoactive intestinal peptide increases VEGF expression to promote proliferation of brain vascular endothelial cells via the 
cAMP/PKA pathway after ischemic insult in vitro. Peptides. 2013;42:105-11.

16. Song Z, Chen Q, Ding Q, Zheng F, Li C, Xu L, Wang H. Function of Ca2+ -/calmodulin-dependent protein kinase IV in Ca2+ -stimulated neuronal signaling and behavior. Sci China Life Sci. 2015;58:613.

17. Yan H, Yuan J, Gao L, Rao J, Hu J. Long noncoding RNA MEG3 activation of p53 mediates ischemic neuronal death in stroke. Neuroscience. 2016;337:191-9.

18. Jiang Y, Yang S, Tao J, Lin Z, Ye X, You Y, Peng J, Hong Z, Chen L. Opposing needling promotes behavior recovery and exerts neuroprotection via the cAMP/PKA/CREB signal transduction pathway in transient MCAO rats. Mol Med Rep. 2016;13:2060-70.

19. Ljubisavljevic MR, Javid A, Oommen J, Parekh K, Nagelkerke N, Shehab S, Adrian TE. The Effects of Different Repetitive Transcranial Magnetic Stimulation (rTMS) Protocols on Cortical Gene Expression in a Rat Model of Cerebral Ischemic-Reperfusion Injury. PLoS One. 2015;10:e0139892.

20. Mesquita RC, Faseyitan OK, Turkeltaub PE, Buckley EM, Thomas A, Kim MN, Durduran T, Greenberg JH, Detre JA, Yodh AG, et al. Blood flow and oxygenation changes due to low-frequency repetitive transcranial magnetic stimulation of the cerebral cortex. J Biomed Opt. 2013;18:067006.

21. Khedr EM, Etraby AE, Hemeda M, Nasef AM, Razek AA. Long-term effect of repetitive transcranial magnetic stimulation on motor function recovery after acute ischemic stroke. Acta Neurol Scand. 2010;121:30-7.

22. Gersner R, Kravetz E, Feil J, Pell G, Zangen A. Long-term effects of repetitive transcranial magnetic stimulation on markers for neuroplasticity: differential outcomes in anesthetized and awake animals. J Neurosci. 2011;31:7521-6.

23. Cha HG, Kim MK. Effects of repetitive transcranial magnetic stimulation on arm function and decreasing unilateral spatial neglect in subacute stroke: a randomized controlled trial. Clin Rehabil. 2016;30:649-56.

24. Ma J, Zhang Z, Kang L, Geng D, Wang Y, Wang M, Cui H. Repetitive transcranial magnetic stimulation (rTMS) influences spatial cognition and modulates hippocampal structural synaptic plasticity in aging mice. Exp Gerontol. 2014;58:256-68.

25. Wang B, Wu N, Liang F, Zhang S, Ni W, Cao Y, Xia D, Xi H. 7,8-dihydroxyflavone, a small-molecule tropomyosin-related kinase $B($ TrkB) agonist, attenuates cerebral ischemia and reperfusion injury in rats. J Mol Histol. 2014;45:129-40.

26. Yang $\mathrm{H}$, Yang L. Targeting cAMP/PKA pathway for glycemic control and type 2 diabetes therapy. $J$ Mol Endocrinol. 2016;57:R93-R108.

27. Lee WR, Shen SC, Wu PR, Chou CL, Shih YH, Yeh CM, Yeh KT, Jiang MC. CSE1L Links CAMP/PKA and Ras/ERK pathways and regulates the expressions and phosphorylations of ERK1/2, CREB, and MITF in melanoma cells. Mol Carcinog. 2016;55:1542-52.

28. Yurimoto S, Fujimoto T, Magari M, Kanayama N, Kobayashi R, Tokumitsu H. In vitro substrate phosphorylation by $\mathrm{Ca}(2)(+) /$ calmodulin-dependent protein kinase kinase using guanosine-5'triphosphate as a phosphate donor. BMC Biochem. 2012;13:27. 
29. Tsokos GC. Calcium signaling in systemic lupus erythematosus lymphocytes and its therapeutic exploitation. Arthritis Rheum. 2008;58:1216-9.

30. Grossinger EM, Kang M, Bouchareychas L, Sarin R, Haudenschild DR, Borodinsky LN, Adamopoulos IE. Ca(2+)-Dependent Regulation of NFATc1 via KCa3.1 in Inflammatory Osteoclastogenesis. J Immunol. 2018;200:749-57.

31. Baek A, Park EJ, Kim SY, Nam BG, Kim JH, Jun SW, Kim SH, Cho SR. High-Frequency Repetitive Magnetic Stimulation Enhances the Expression of Brain-Derived Neurotrophic Factor Through Activation of $\mathrm{Ca}(2+)$-Calmodulin-Dependent Protein Kinase II-cAMP-Response Element-Binding Protein Pathway. Front Neurol. 2018;9:285.

32. Harrison BJ, Flight RM, Gomes C, Venkat G, Ellis SR, Sankar U, Twiss JL, Rouchka EC, Petruska JC. IB4-binding sensory neurons in the adult rat express a novel 3' UTR-extended isoform of CaMK4 that is associated with its localization to axons. J Comp Neurol. 2014;522:308-36.

33. Ye X, Yu L, Zuo D, Zhang L, Zu J, Hu J, Tang J, Bao L, Cui C, Zhang R, et al. Activated mGluR5 protects BV2 cells against $O G D / R$ induced cytotoxicity by modulating BDNF-TrkB pathway. Neurosci Lett. 2017;654:70-9.

34. Wang HY, Crupi D, Liu J, Stucky A, Cruciata G, Di Rocco A, Friedman E, Quartarone A, Ghilardi MF. Repetitive transcranial magnetic stimulation enhances BDNF-TrkB signaling in both brain and lymphocyte. J Neurosci. 2011;31:11044-54.

35. Lin WY, Chang YC, Lee HT, Huang CC. CREB activation in the rapid, intermediate, and delayed ischemic preconditioning against hypoxic-ischemia in neonatal rat. J Neurochem. 2009;108:847-59.

\section{Tables}

Table 1. Primer sequence for reverse transcription-quantitative polymerase chain reaction

\begin{tabular}{ll}
\hline Genes & Sequences (5'-3') \\
\hline TrkB & F: 5'-TCGGATACAAGAAGGACAAC-3' \\
& R: 5'-CTGCATTGGGACAGAGTAG-3' \\
\multirow{2}{*}{-actin } & F: 5'-GTAAAGACCTCTATGCCAACA-3' \\
& R: 5'-GGACTCATCCTACTCCTGCT-3' \\
\hline
\end{tabular}

Note: F, forward; R, reverse; TrkB, tropomyosin receptor kinase B.

Table 2. Neurological function in rats after MCAO modeling 


\begin{tabular}{|c|c|c|c|c|}
\hline Index & n Time & Mock & Sham & MCAO \\
\hline \multirow[t]{4}{*}{ Neurological deficit score } & $51 \mathrm{~d}$ & $0.00 \pm 0.00$ & $0.00 \pm 0.00$ & $2.80 \pm 0.45^{*}$ \\
\hline & $3 d$ & $0.20 \pm 0.45$ & $0.20 \pm 0.45$ & $2.20 \pm 0.45^{*}$ \\
\hline & $7 \mathrm{~d}$ & $0.20 \pm 0.45$ & $0.00 \pm 0.00$ & $1.80 \pm 0.45^{*}$ \\
\hline & $14 \mathrm{~d}$ & $0.00 \pm 0.00$ & $0.00 \pm 0.00$ & $1.60 \pm 0.55^{*}$ \\
\hline \multirow[t]{4}{*}{ Wire grasping ability score 5} & $51 \mathrm{~d}$ & $2.80 \pm 0.45$ & $3.00 \pm 0.18$ & $0.20 \pm 0.45^{*}$ \\
\hline & $3 d$ & $2.80 \pm 0.45$ & $2.60 \pm 0.55$ & $0.40 \pm 0.55^{*}$ \\
\hline & $7 \mathrm{~d}$ & $2.80 \pm 0.45$ & $2.60 \pm 0.55$ & $0.80 \pm 0.45^{*}$ \\
\hline & $14 \mathrm{~d}$ & $2.60 \pm 0.55$ & $2.80 \pm 0.45$ & $1.20 \pm 0.45^{*}$ \\
\hline \multirow[t]{4}{*}{ The right reaction times 5} & $51 \mathrm{~d}$ & $15.20 \pm 2.77$ & $14.80 \pm 2.28$ & $81.60 \pm 0.89 *$ \\
\hline & $3 d$ & $15.00 \pm 1.58$ & $15.00 \pm 1.58$ & $82.40 \pm 1.34^{*}$ \\
\hline & $7 d$ & $14.60 \pm 1.34$ & $14.40 \pm 3.58$ & $33.20 \pm 1.10^{*}$ \\
\hline & $14 \mathrm{~d}$ & $14.20 \pm 0.84$ & $13.80 \pm 1.92$ & $23.80 \pm 0.45^{*}$ \\
\hline
\end{tabular}

Notes: Table 1 shows the comparison of neurological deficits, motion balance, and learning and memory functions in rats among different groups. ${ }^{*} p<0.05 \mathrm{vs}$. the sham-operated rats. The above measurement data were expressed as mean \pm standard deviation. The repeated measurement analysis of variance was applied for the comparison of data at different time points, and Tukey's test was utilized for post hoc test. $n=5$. MCAO, middle cerebral artery occlusion.

Table 3. Neurological function in rats after MCAO modeling and stimulation with $1 \mathrm{~Hz}$ or $10 \mathrm{~Hz}$ rTMS

\begin{tabular}{cccccc}
\hline Index & nTime & Sham & MCAO & \multicolumn{2}{c}{ MCAO + 1 Hz MCAO + 10 Hz } \\
\hline Neurological deficit score 5 & $1 \mathrm{~d}$ & $0.00 \pm 0.00$ & $2.80 \pm 0.45$ & $2.00 \pm 0.71^{*}$ & $1.60 \pm 0.55^{*}$ \\
\cline { 2 - 6 } & $3 \mathrm{~d}$ & $0.20 \pm 0.45$ & $2.40 \pm 0.55$ & $1.60 \pm 0.55^{*}$ & $1.20 \pm 0.45^{*}$ \\
\cline { 2 - 6 } & $7 \mathrm{~d}$ & $0.00 \pm 0.00$ & $1.80 \pm 0.45$ & $1.40 \pm 0.55^{*}$ & $0.80 \pm 0.45^{*}$ \\
\cline { 2 - 6 } & $14 \mathrm{~d}$ & $0.20 \pm 0.45$ & $1.40 \pm 0.55$ & $1.00 \pm 0.71^{*}$ & $0.60 \pm 0.45^{*}$ \\
\hline Wire grasping ability score 5 & $1 \mathrm{~d}$ & $2.80 \pm 0.45$ & $0.20 \pm 0.45$ & $1.20 \pm 0.45^{*}$ & $1.40 \pm 0.55^{*}$ \\
\cline { 2 - 6 } & $3 \mathrm{~d}$ & $2.80 \pm 0.45$ & $0.40 \pm 0.55$ & $1.60 \pm 0.55^{*}$ & $1.80 \pm 0.45^{*}$ \\
\cline { 2 - 6 } & $7 \mathrm{~d}$ & $2.60 \pm 0.55$ & $0.80 \pm 0.84$ & $1.80 \pm 0.45^{*}$ & $2.00 \pm 0.71^{*}$ \\
\cline { 2 - 6 } The right reaction times & $14 \mathrm{~d}$ & $2.80 \pm 0.45$ & $1.20 \pm 0.45$ & $2.00 \pm 0.71^{*}$ & $2.40 \pm 0.55^{*}$ \\
\hline & $1 \mathrm{~d}$ & $15.40 \pm 2.70$ & $1.80 \pm 0.84$ & $2.60 \pm 0.89^{*}$ & $5.40 \pm 1.14^{*}$ \\
\cline { 2 - 6 } & $3 \mathrm{~d}$ & $14.60 \pm 2.07$ & $2.60 \pm 1.14$ & $4.00 \pm 1.85^{*}$ & $6.40 \pm 2.61^{*}$ \\
\cline { 2 - 6 } & $7 \mathrm{~d}$ & $14.20 \pm 1.92$ & $3.00 \pm 1.00$ & $6.60 \pm 1.52^{*}$ & $11.20 \pm 1.64^{*}$ \\
\cline { 2 - 5 } & $14 \mathrm{~d}$ & $14.20 \pm 0.69^{*} 3.80 \pm 0.84$ & $7.40 \pm 0.89^{*}$ & $13.00 \pm 1.87^{*}$ \\
\hline
\end{tabular}

Notes: Table 2 shows the comparison of neurological deficits, motion balance, and learning and memory functions in rats stimulated by rTMS. $* p<0.05$ vs. the MCAO rats. The above measurement data are expressed as mean \pm standard deviation. The repeated measurement analysis of variance was applied for the comparison of data at different time points, Tukey's test was utilized for post hoc test, $n=5$. MCAO, middle cerebral artery occlusion. 
Figures
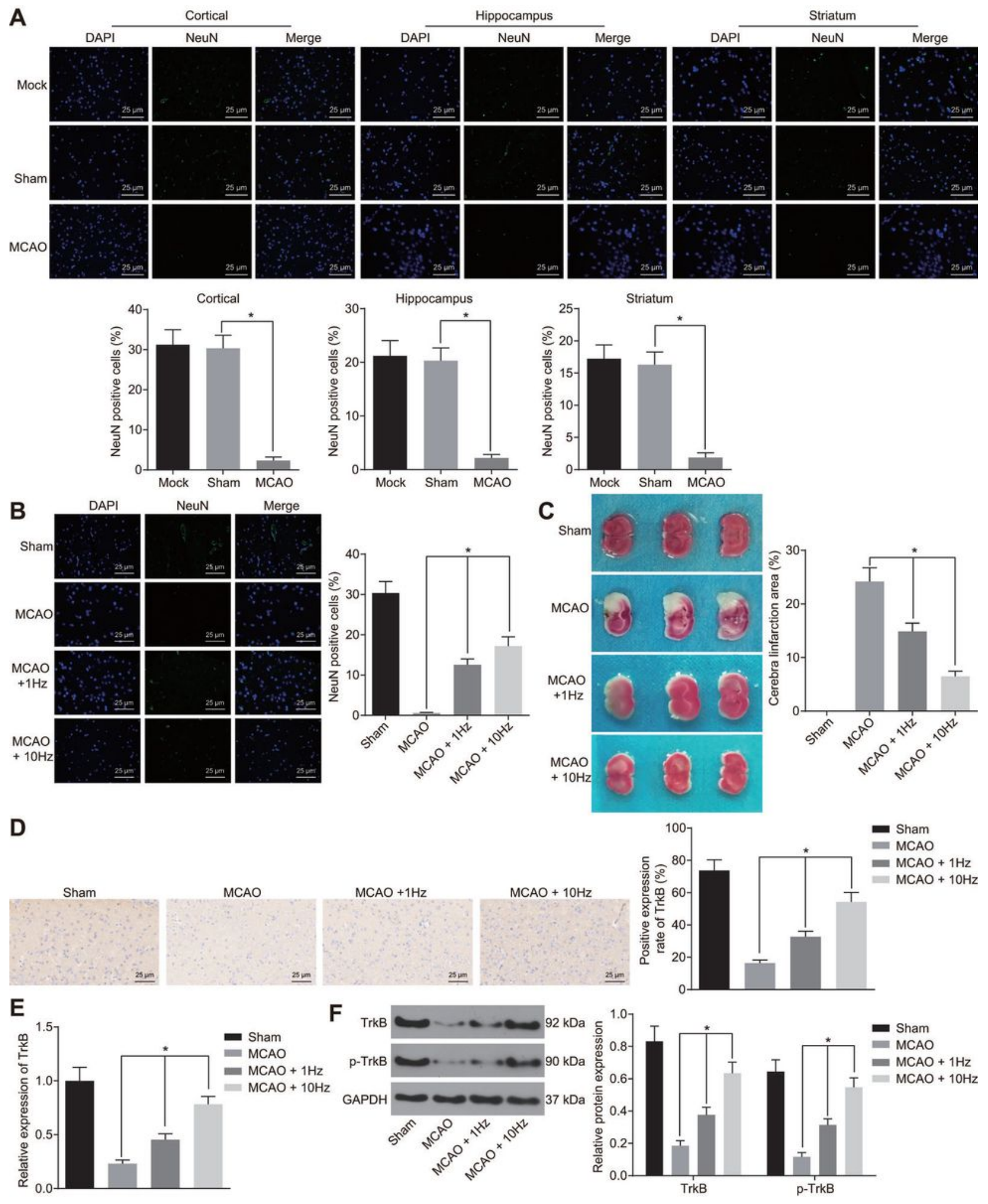

Figure 1 
The nerve recovery ability is promoted and TrkB expression is increased by rTMS in cerebral ischemia rats. A), The immunofluorescence staining of NeuN in the cortex, hippocampus and striatum of rats after MCAO modeling $(\times 400) .{ }^{*} p<0.05$ vs. the sham-operated rats. B), The immunofluorescence staining of NeuN in the cortex of MCAO rats under rTMS stimulation ( $\times 400)$. C), Representative images of cerebral infarction in the rats and the percentage of cerebral infarction area in the cortex of MCAO rats under rTMS stimulation measured by TTC staining. ${ }^{*} p<0.05$ vs. the MCAO rats. D), The positive expression of TrkB in the cerebral cortical tissues of MCAO rats under rTMS stimulation detected by immunofluorescence staining $(\times 400) .{ }^{*} p<0.05$ vs. the MCAO rats. E), The mRNA expression of TrkB in the cortex of MCAO rats under rTMS stimulation determined by RT-qPCR. ${ }^{*} p<0.05$ vs. the MCAO rats. F), The TrkB protein expression and its phosphorylation in the cortex of MCAO rats under rTMS stimulation determined by Western blot analysis. ${ }^{*} p<0.05$ vs. the MCAO rats. The above data were measurement data and expressed as mean \pm standard deviation. One-way analysis of variance was used for comparisons among multiple groups, followed by Tukey's post hoc test. $n=5$. rTMS, repetitive transcranial magnetic stimulation; TrkB, tropomyosin receptor kinase B; NeuN, neuronal nuclei; TTC, Triphenyltetrazolium chloride; MCAO, middle cerebral artery occlusion; RT-qPCR, reverse transcription quantitative polymerase chain reaction. 


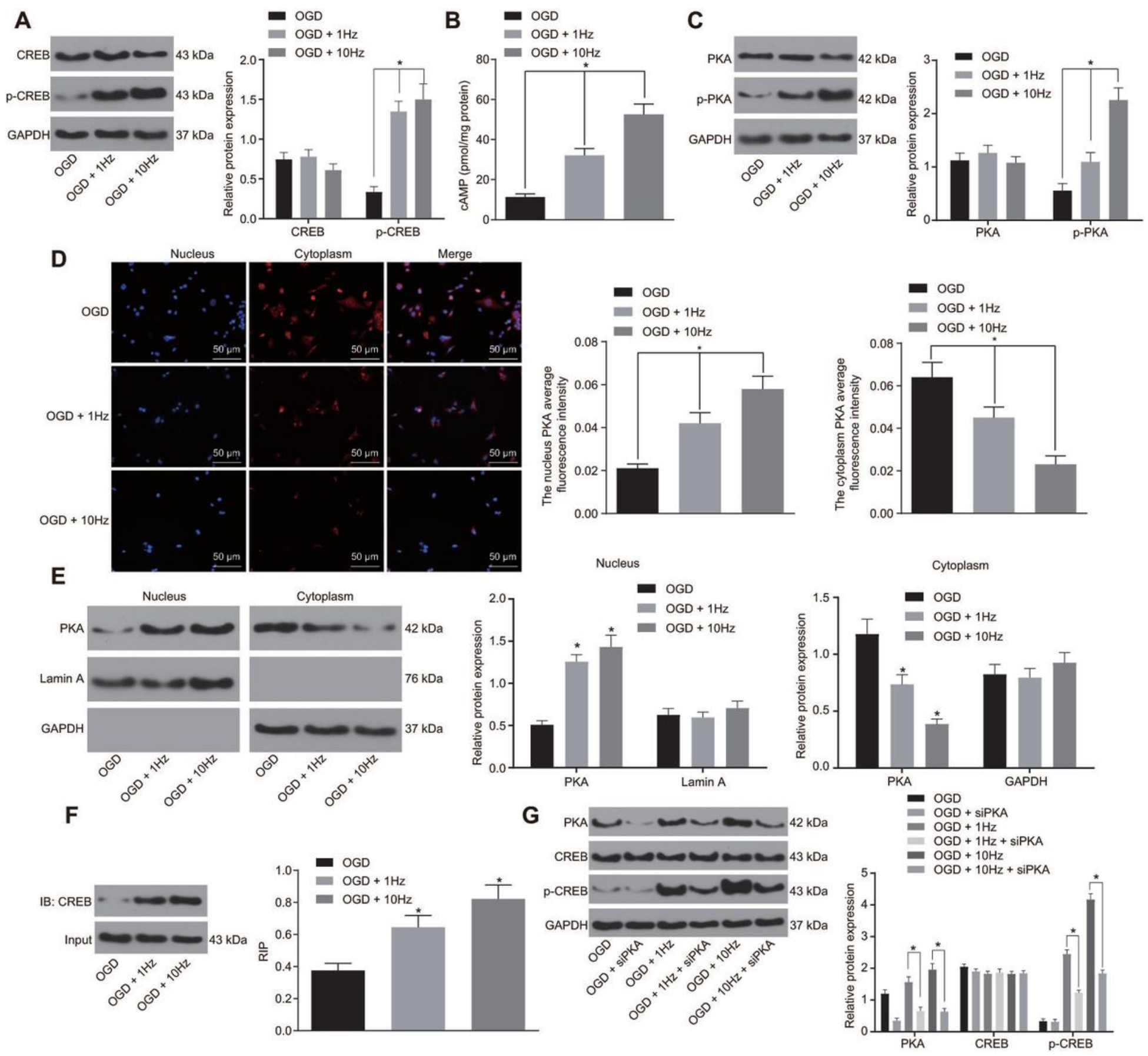

Figure 2

2 rTMS induces the activation of CREB by promoting the cAMP/PKA pathway. A), The expression of CREB and its phosphorylation level in OGD-treated neurons after stimulation of $1 \mathrm{~Hz}$ or $10 \mathrm{~Hz} \mathrm{rTMS}$ measured by Western blot analysis. ${ }^{*}<0.05$ vs. the OGD-treated neurons. B), The expression of PKA and its phosphorylation level determined by Western blot analysis. ${ }^{*} p<0.05$ vs. the OGD-treated neurons. C), The expression of cAMP tested by ELISA. * $p<0.05$ vs. the OGD-treated neurons. D), Subcellular localization analysis of PKA performed by immunofluorescence $(\times 200) .{ }^{*} p<0.05$ vs. the OGD-treated neurons. E), The expression of PKA, GAPDH and Lamin A in the nucleus and cytoplasm measured by Western blot analysis. F), The binding relationship between PKA and CREB verified by coimmunoprecipitation and Western blot analysis. G), The expression of CREB and its phosphorylation level 
after inhibition of PKA determined by Western blot analysis. ${ }^{*} p<0.05$ vs. the OGD-treated neurons stimulated with $1 \mathrm{~Hz}$ rTMS, \# $\mathrm{p}<0.05$ vs. the OGD-treated neurons stimulated with $10 \mathrm{~Hz}$ rTMS. The above data were measurement data and expressed as mean \pm standard deviation. One-way analysis of variance was used for comparison among multiple groups, followed by Tukey's post hoc test. $n=5$. rTMS, repetitive transcranial magnetic stimulation; CREB, CAMP-response element binding protein; PKA, protein kinase A; OGD, oxygen-glucose deprivation; ELISA, Enzyme-linked immunoassay; GAPDH, glyceraldehyde-3-phosphate dehydrogenase.

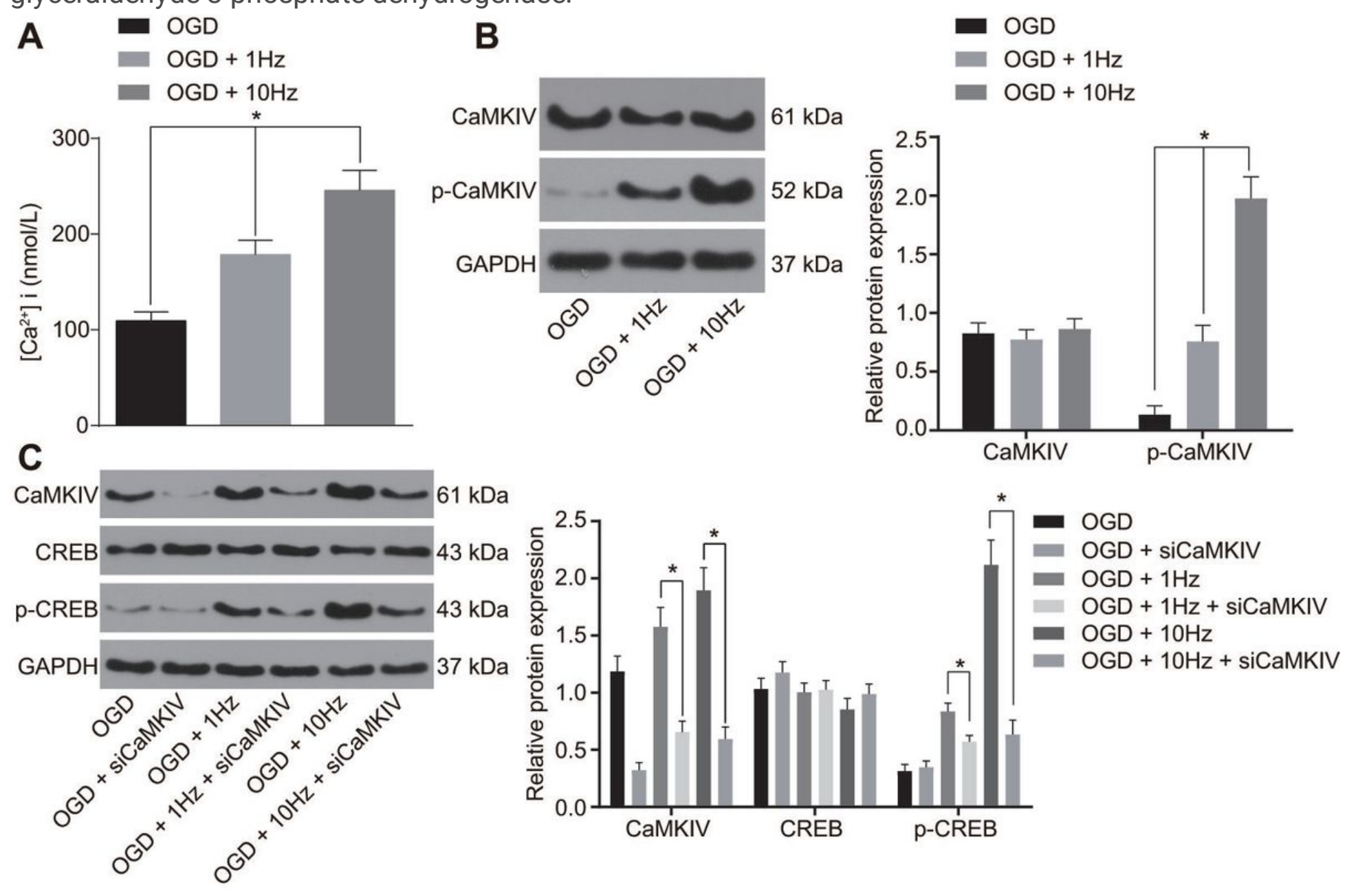

\section{Figure 3}

rTMS activates CREB by inducing activation of the $\mathrm{Ca} 2+$ /CaMKIV pathway. A), The intracellular $\mathrm{Ca} 2+$ concentration measured using fluorescence probe Fura- $2{ }^{*} p<0.05$ vs. the OGD-treated neurons. B), The expression of CaMKIV and its phosphorylation level measured by western blot analysis, ${ }^{*}<0.05$ vs. the OGD-treated neurons. C), The expression of CREB and its phosphorylation after downregulation of CaMKIV determined by Western blot analysis, * $p<0.05$ vs. the OGD-treated neurons stimulated with $1 \mathrm{~Hz}$ rTMS, \# $p<0.05$ vs. the OGD-treated neurons stimulated with $10 \mathrm{~Hz}$ rTMS. The above data were measurement data and expressed as mean \pm standard deviation. One-way analysis of variance was used for comparison among multiple groups, followed by Tukey's post hoc test, $n=5$. rTMS, repetitive transcranial magnetic stimulation; CREB, CAMP-response element binding protein; OGD, oxygen-glucose deprivation. 


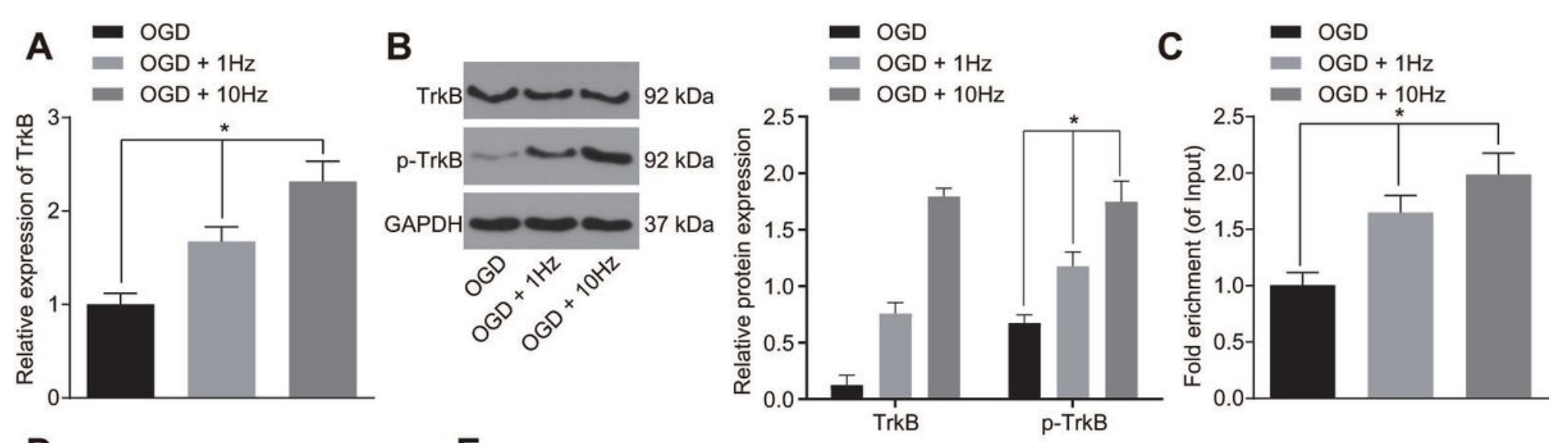

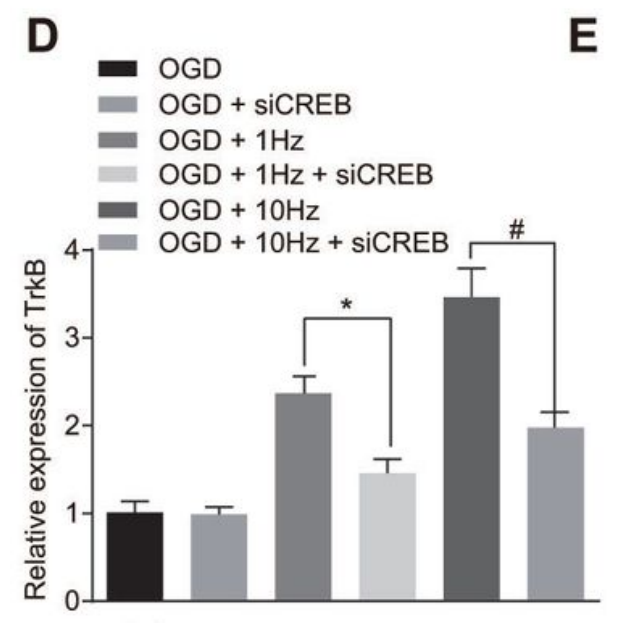

$\mathbf{F}$

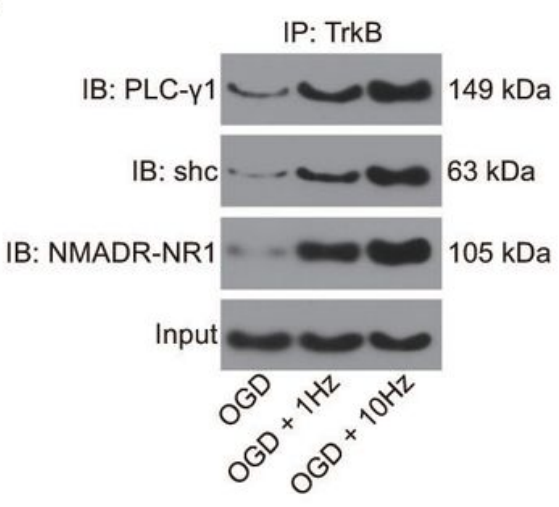

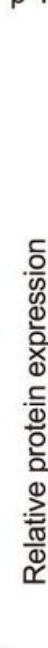
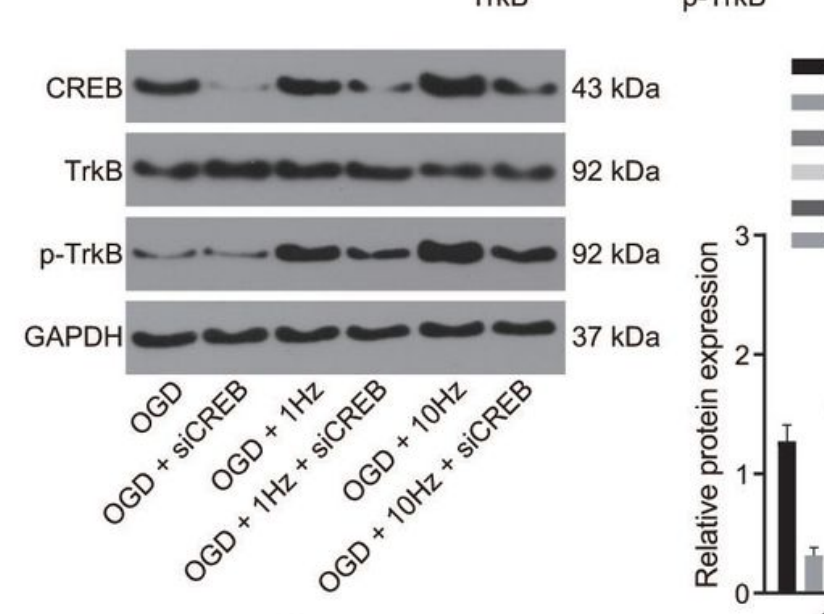

G

IP: TrkB

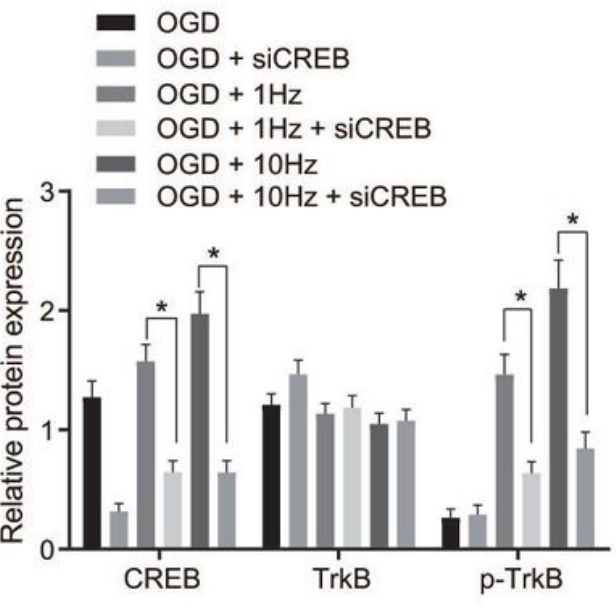

IB: PLC-y1 149 kDa

IB: shc $63 \mathrm{kDa}$
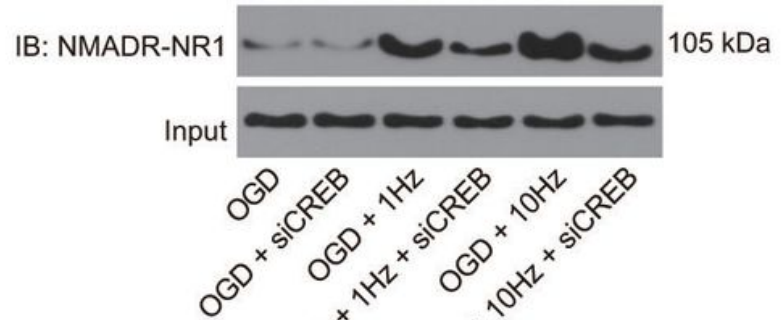

$\mathrm{COC}^{\mathrm{cos} x}$

\section{Figure 4}

4 rTMS activates CREB to upregulate TrkB. A), The mRNA expression of TrkB determined by RT-qPCR, * $p$ $<0.05$ vs. the OGD-treated neurons. B), The expression of TrkB and its phosphorylation level determined by Western blot analysis, ${ }^{*} p<0.05$ vs. the OGD-treated neurons. C), The binding of CREB to TrkB at CRE sites detected by ChIP, ${ }^{\star} p<0.05$ vs. the OGD-treated neurons. D), The mRNA expression of TrkB determined by RT-qPCR after CREB is down-regulated, ${ }^{*} p<0.05$ vs. the OGD-treated neurons stimulated with $1 \mathrm{~Hz}$ rTMS, \# $\mathrm{p}<0.05$ vs. the OGD-treated neurons stimulated with $10 \mathrm{~Hz}$ rTMS. E), The expression of TrkB and its phosphorylation level determined by Western blot analysis after CREB was downregulated, ${ }^{*} p<0.05$ vs. the OGD-treated neurons stimulated with $1 \mathrm{~Hz}$ rTMS, $\# p<0.05$ vs. the OGDtreated neurons stimulated with $10 \mathrm{~Hz}$ rTMS. F), The expression of PLC- $\gamma 1$, SHC and NMADR-NR1 co- 
immunoprecipitated with TrkB measured by Western blot analysis, ${ }^{*} p<0.05$ vs. the OGD-treated neurons. G), The expression of PLC- $y 1$, SHC and NMADR-NR1 co-immunoprecipitated with TrkB measured by Western blot analysis after downregulation of CREB, ${ }^{*} p<0.05$ vs. the OGD-treated neurons stimulated with $1 \mathrm{~Hz}$ rTMS, \# $\mathrm{p}<0.05$ vs. the OGD-treated neurons stimulated with $10 \mathrm{~Hz}$ rTMS. The above data were measurement data and expressed as mean \pm standard deviation. One-way analysis of variance was used for comparison among multiple groups, followed by Tukey's post hoc test, $n=5$. rTMS, repetitive transcranial magnetic stimulation; TrkB, tropomyosin receptor kinase B; CREB, CAMP-response element binding protein; RT-qPCR, reverse transcription quantitative polymerase chain reaction; OGD, oxygenglucose deprivation.
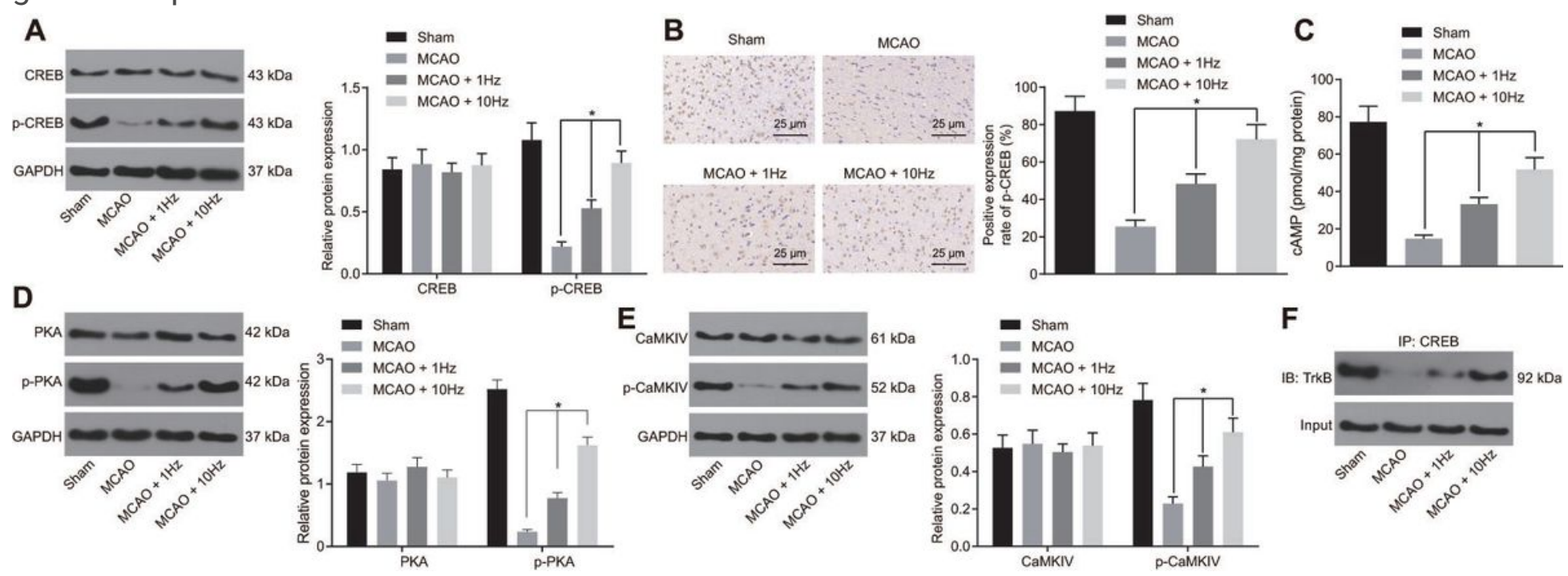

Figure 5

rTMS strengthens the binding of CREB to TrkB in vivo. A), The expression of CREB and its phosphorylation level determined by Western blot analysis, ${ }^{*} p<0.05$ vs. the MCAO rats. $\left.B\right)$, The positive rate of phosphorylated CREB protein determined by immunohistochemistry $(\times 400)$, ${ }^{*} p<0.05 \mathrm{vs}$. the MCAO rats. C), The expression of CAMP tested by ELISA, ${ }^{*} p<0.05$ vs. the MCAO rats. D), The expression of PKA and its phosphorylation level determined by Western blot analysis, ${ }^{*} p<0.05$ vs. the MCAO rats. $E)$, The expression of CaMKIV and its phosphorylation level determined by Western blot analysis, * $p<$ 0.05 vs. the MCAO rats. F), The binding of CREB to TrkB determined by co-immunoprecipitation and the expression of TrkB co-immunoprecipitated with CREB measured by Western blot analysis, * $p<0.05$ vs. the MCAO rats. The above data were measurement data and expressed as mean \pm standard deviation. One-way analysis of variance was used for comparison among multiple groups, followed by Tukey's post hoc test, $n=5$. CREB, cAMP-response element binding protein; MCAO, middle cerebral artery occlusion; ELISA, Enzyme-linked immunoassay; PKA, protein kinase A; TrkB, tropomyosin receptor kinase B. 


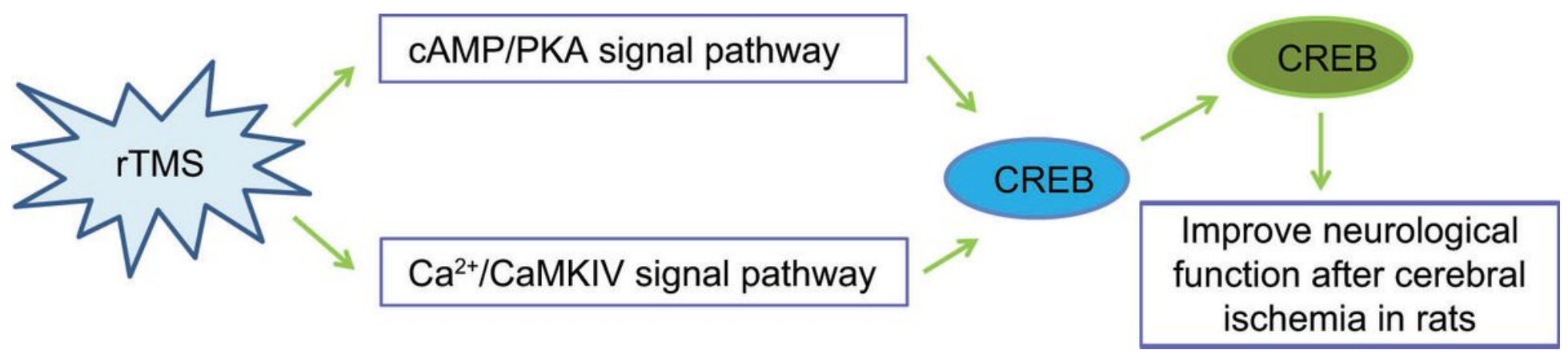

Figure 6

The molecular mechanism of rTMS in cerebral ischemia. rTMS activates CREB through the CAMP/PKA pathway and $\mathrm{Ca} 2+/ \mathrm{CaMKIV}$ pathway, thereby improving neurological function in rats with cerebral ischemia.

\section{Supplementary Files}

This is a list of supplementary files associated with this preprint. Click to download.

- SupplementaryFigure1.eps 